\title{
Optimizing the diagnostic workup of infective endocarditis: An urgent need for studies focused on defining the decision-making process
}

\author{
María Nazarena Pizzi, MD, a and Nuria Fernández-Hidalgo, MD, PhD ${ }^{b}$ \\ a Department of Cardiology, Hospital Universitari Vall d'Hebron, Vall d'Hebron Institut de \\ Recerca (VHIR), Universitat Autònoma de Barcelona, Barcelona, Spain \\ b Servei de Malalties Infeccioses, Hospital Universitari Vall d'Hebron, Vall d'Hebron Institut de \\ Recerca (VHIR), Universitat Autònoma de Barcelona, Barcelona, Spain
}

Received Aug 29, 2018; accepted Aug 29, 2018

doi:10.1007/s12350-018-1434-1

\section{See related article, pp. 592-608}

For decades, the modified Duke criteria ${ }^{1}$ have been widely used in the diagnosis of infective endocarditis (IE). Nonetheless, because of the technical limitations of echocardiography, the sensitivity and specificity of the Duke criteria are low in the growing percentage of complex IE patients with repaired congenital heart diseases, prosthetic tubes, valves, and other devices.

To overcome this limitation, the 2015 IE guidelines of the European Society of Cardiology (ESC) incorporated imaging tests other than echocardiography (cardiac CT, FDG-PET/CT, and radiolabeled leukocyte SPECT/ $\mathrm{CT}$ ) in the diagnostic process (multimodal imaging approach), considering the findings as major diagnostic criteria of IE. $^{2}$ In addition, these guidelines strongly encourage management of IE patients in specialized units and enhancing the role of expert cardiac imaging. However, the guidelines do not provide a clear algorithm defining the need, sequence, or timing of these tests. This lack of an endorsed algorithm has compelled IE unit specialists to develop local strategies for clinical decision-making, and these may have certain

Reprint requests: María Nazarena Pizzi, MD, Department of Cardiology, Hospital Universitari Vall d'Hebron, Vall d'Hebron Institut de Recerca (VHIR), Universitat Autònoma de Barcelona, Passeig de la Vall d'Hebron 119-129, 08035 Barcelona, Spain; nachi_pizzi@yahoo.com.ar

J Nucl Cardiol 2020;27:609-11.

$1071-3581 / \$ 34.00$

Copyright (C) 2018 American Society of Nuclear Cardiology. limitations: (1) They may be focused on center-based practice according to available facilities, and therefore poorly generalizable; (2) Their cost-effectiveness may not be proven; and (3) They may place excessive importance on detection of lesions without significant clinical relevance.

In this context, the attempt by Anna Gomes et al. to provide a systematic approach to the diagnosis of IE published in this issue of the Journal of Nuclear Cardiology is a welcome effort. ${ }^{3}$ The authors evaluated the single-center adherence to a diagnostic flowchart previously reported by the same group, ${ }^{4}$ and re-assessed the usefulness of multidetector computed tomography angiography (MDCTA) and FDG-PET/CT in the diagnosis of IE. However, although the objectives are clear, to our mind, the contributions of this study are rather limited.

The algorithm presented is complex and may not be easily applicable in other centers. Not all hospitals possess the technology described, and even in those where it is available, a highly specialized professional team with experience in the diagnosis of IE is needed to properly interpret the imaging findings. Furthermore, implementation of this algorithm requires the full knowledge and commitment of all professionals involved in managing these patients, as well as the hospital financial managers, who must agree with the cost implied. In this sense, it is significant that patient management in the author's hospital did not comply with their own protocol in $31 \%$ of cases, as was reported in the study. In addition, although the results of the study are in accordance with the literature, the added value of MDCTA and FDG-PET/CT to the modified Duke criteria and their accuracy in the diagnosis of IE have been 
amply demonstrated in previous studies with larger samples. $^{5-7}$

The relatively low percentage (68\%) of patients with suspected endocarditis on prosthetic material in whom transesophageal echocardiography (TEE) was performed is quite surprising. Despite its limitations, echocardiography remains a fundamental test in the initial assessment of patients with suspected IE. The value of TEE for this purpose is thoroughly established and unquestionable in all IE-related clinical scenarios with the exception of right-sided IE. In device-related IE, TEE enables examination of the distal part of the superior vena cava and improves detection of intracardiac vegetations. ${ }^{2}$

Since FDG-PET/CT was incorporated in the diagnostic process of IE, we have learned that the contribution of this test in suspected IE should be analyzed separately depending on whether the infection involves a prosthetic valve or a device. In their study, the authors show the results according to whether the suspected IE was related to a native valve or prosthetic material, but they did not differentiate between prosthetic valves and devices. This is relevant, as the diagnostic process differs in these two infections.

We also noted that the radiologic images obtained were evaluated blindly. This would make sense in an experimental design, but not in daily clinical practice. Image experts should be involved in the entire diagnostic process of IE, from the time the infection is suspected up to establishment of the definite diagnosis, because in daily practice, the clinical information (pretest probability) can modify interpretation of the images. In our experience, one of the cornerstones of the learning curve in image interpretation is the continuous feedback between clinicians and image specialists in each and every case of suspected IE.

In the proposed algorithm, FDG-PET/CT is mainly indicated to investigate the presence of septic metastasis once the IE diagnosis has been established. Specifically, FDG-PET/CT is used to visualize peripheral findings in 5 of 7 of the suggested indications for this technique. However, despite the importance the authors place on the search for septic embolisms, we were unable to find a description of the septic metastases found on FDG$\mathrm{PET} / \mathrm{CT}$ in the article. It would have been interesting to provide these data to support their hypothesis, and to know whether the embolisms were symptomatic or not, and whether their presence modified the patients' management in any way.

FDG-PET/CT is a whole-body examination with a high, well-documented sensitivity for detecting peripheral lesions ${ }^{8,9}$; hence, physicians may be tempted to use it with this diagnostic objective. In the 2015 ESC guidelines, screening for silent embolisms is encouraged to add a minor criterion when the diagnosis of IE is not definitive. However, it is essential to clarify whether the importance of this imaging test lies in the mere detection of silent lesions or in its ability to provide findings that are relevant for clinical decision-making. We must bear in mind that an excess of information can sometimes lead to inappropriate decisions.

We believe that the main value of FDG-PET/CT in prosthetic valve IE is to provide an early diagnosis of the valve involvement when the initial echocardiography findings are negative or inconclusive, not for the detection of septic embolisms. It is at this time (before the anatomical lesion is evident) when FDG-PET/CT is most valuable, as we can make relevant decisions (e.g., discharge without antibiotics or urgent surgery) based on the findings. In native valve IE, echocardiography usually has no difficulty in establishing the diagnosis. In this setting, the true sensitivity of FDG-PET/CT for cardiac diagnoses is practically unknown, as the results with this test are based on small series included in studies performed with other objectives. ${ }^{10,11}$ However, as an expert opinion that must be evaluated in further studies, we believe that FDG-PET/CT in combination with MDCTA (FDG-PET/CTA) could be successful for depicting perivalvular complications in cases with an inconclusive echocardiography study.

In both prosthetic and native valve IE, if there is no valve lesion, the finding of peripheral embolisms is highly unlikely. If there is a valve lesion, a systematic search for asymptomatic peripheral embolisms does not usually modify the management of the infection, as lesions that require a change of approach (e.g., draining a collection or prolonging antibiotic treatment) are usually symptomatic, eliciting pain, fever, or neurological signs. If the diagnostic process is properly performed, the need to add an embolic lesion as a minor criterion to reach a definite diagnosis rarely occurs; hence, there are few situations that justify performing FDG-PET/CT scanning exclusively for this objective.

The population at risk of IE is becoming increasingly older and frailer, and it is not intrinsically good to perform many imaging tests. We have to consider the potential complications related to (unnecessary) insertion of intravascular catheters, administration of intravenous contrast, and radiation exposure. And, as was mentioned above, an excess of information can lead to confusion. For example, the finding of a mycotic cerebral aneurysm in an asymptomatic patient could lead to a delay in cardiac surgery to treat severe valve regurgitation and heart failure. ${ }^{12}$ In our opinion, if a finding will not improve decision-making, it is better not to look for it.

Despite the limitations mentioned, this study has some positive aspects to be highlighted. First, FDG- 
PET/CT was performed promptly, between 4 and 7 days after the start of antibiotic treatment. To our mind, this is the time when it makes sense to use FDG-PET/CT, as it can detect infection at the valve early, before the anatomical lesion develops. Second, FDG-PET/CT study was not limited to episodes occurring a certain time after surgery. The 2015 ESC guidelines state that FDG-PET/CT would not be advisable before a safety period of three post-operative months because it might yield false-positive results related to post-operative inflammation. ${ }^{2}$ This recommendation, although reasonable in light of the lack of data, is not supported by scientific evidence, and recent studies are suggesting that FDG-PET/CT findings would not be specifically affected by the postoperative time when the examination is performed. ${ }^{7}$

We are faced with the challenge of attending a growing number of complex patients with limited resources. Although the availability of imaging tests is also increasing, we must make rational use of these complementary examinations. Performing a large number of tests does not always result in better management. On the contrary, it can confuse, increase health system costs, and even worse, imply a personal cost for the patient. At a time when more studies are published than we can handle, we need solid evidence to help us make decisions in this increasingly more complex patient population.

\section{Acknowledgements}

We thank Celine Cavallo for language support.

\section{Disclosures}

María Nazarena Pizzi and Nuria Fernández-Hidalgo have nothing to disclose.

\section{References}

1. Li JS, Sexton DJ, Mick N, Nettles R, Fowler VG Jr, Ryan T, et al. Proposed modifications to the Duke criteria for the diagnosis of infective endocarditis. Clin Infect Dis 2000;30:633-8.
2. Habib G, Lancellotti P, Antunes MJ, Bongiorni MG, Casalta JP, Del Zotti F, et al. 2015 ESC guidelines for the management of infective endocarditis: The Task Force for the Management of Infective Endocarditis of the European Society of Cardiology (ESC). Endorsed by: European Association for Cardio-Thoracic Surgery (EACTS), the European Association of Nuclear Medicine (EANM). Eur Heart J 2015;36:3075-128.

3. Gomes A, van Geel PP, Santing M, Prakken NHJ, Ruis ML, van Assen S, et al. Imaging infective endocarditis: Adherence to a diagnostic flowchart and direct comparison of imaging techniques. J Nucl Cardiol 2018. https://doi.org/10.1007/s12350-018-1383-8.

4. Gomes A, Glaudemans AWJM, Touw DJ, van Melle JP, Willems TP, Maass AH, et al. Diagnostic value of imaging in infective endocarditis: a systematic review. Lancet Infect Dis 2017;17:e114.

5. Saby L, Laas O, Habib G, Cammilleri S, Mancini J, Tessonnier L, et al. Positron emission tomography/computed tomography for diagnosis of prosthetic valve endocarditis: Increased valvular ${ }^{18} \mathrm{~F}$ fluorodeoxyglucose uptake as a novel major criterion. J Am Coll Cardiol 2013;61:2374-82.

6. Pizzi MN, Roque A, Fernández-Hidalgo N, Cuéllar-Calabria H, Ferreira-González I, Gonzàlez-Alujas MT, et al. Improving the diagnosis of infective endocarditis in prosthetic valves and intracardiac devices with ${ }^{18} \mathrm{~F}$-fluordeoxyglucose positron emission tomography/computed tomography angiography: initial results at an infective endocarditis referral center. Circulation 2015;132:1113-26.

7. Swart LE, Gomes A, Scholtens AM, Sinha B, Tanis W, Lam $\mathrm{MGEH}$, et al. Improving the diagnostic performance of ${ }^{18} \mathrm{~F}$-FDG PET/CT in prosthetic heart valve endocarditis. Circulation 2018. https://doi.org/10.1161/CIRCULATIONAHA.118.035032.

8. Van Riet J, Hill EE, Gheysens O, et al. ${ }^{18}$ F-FDG PET/CT for early detection of embolism and metastatic infection in patients with infective endocarditis. Eur $\mathbf{J}$ Nucl Med Mol Imaging 2010;37:1189-97.

9. Bonfiglioli R, Nanni C, Morigi JJ, et al. ${ }^{18}$ F-FDG PET/CT diagnosis of unexpected extracardiac septic embolisms in patients with suspected cardiac endocarditis. Eur J Nucl Med Mol Imaging 2013;40:1190-6.

10. Kestler M, Muñoz P, Rodríguez-Créixems M, Collaboration with the Group for the Management of Infectious Endocarditis (GAME), et al. Role of ${ }^{18} \mathrm{~F}$-FDG PET in patients with infectious endocarditis. J Nucl Med 2014;55:1-6.

11. Granados U, Fuster D, Pericas JM, et al. Diagnostic accuracy of ${ }^{18} \mathrm{~F}$-FDG PET/CT in infective endocarditis and implantable cardiac electronic device infection: a cross-sectional study. J Nucl Med 2016;57:1726-32.

12. Duval X, Iung B, Klein I, Brochet E, Thabut G, Arnoult F, et al. Effect of early cerebral magnetic resonance imaging on clinical decisions in infective endocarditis: a prospective study. Ann Intern Med 2010;152:497-504, W175. 